\title{
ON THE PRINCIPAL EIGENCURVE OF THE $p$-LAPLACIAN RELATED TO THE SOBOLEV TRACE EMBEDDING
}

Abstract. We prove that for any $\lambda \in \mathbb{R}$, there is an increasing sequence of eigenvalues $\mu_{n}(\lambda)$ for the nonlinear boundary value problem

$$
\begin{cases}\Delta_{p} u=|u|^{p-2} u & \text { in } \Omega, \\ |\nabla u|^{p-2} \partial u / \partial \nu=\lambda \varrho(x)|u|^{p-2} u+\mu|u|^{p-2} u & \text { on } \partial \Omega,\end{cases}
$$

and we show that the first one $\mu_{1}(\lambda)$ is simple and isolated; we also prove some results about variations of the density $\varrho$ and the continuity with respect to the parameter $\lambda$.

1. Introduction and notations. Let $\Omega$ be a smooth bounded domain in $\mathbb{R}^{N} ; N \geq 1 ; 1<p<\infty$ and $\varrho \in L^{\infty}(\partial \Omega)$ with $\varrho \not \equiv 0$ which can change the sign; $\lambda, \mu \in \mathbb{R}$. We consider the following nonlinear boundary value problem:

$$
\begin{gathered}
\Delta_{p} u=|u|^{p-2} u \quad \text { in } \Omega, \\
|\nabla u|^{p-2} \frac{\partial u}{\partial \nu}=\lambda \varrho(x)|u|^{p-2} u+\mu|u|^{p-2} u \quad \text { on } \partial \Omega .
\end{gathered}
$$

The $p$-Laplacian $\Delta_{p} u=\nabla \cdot\left(|\nabla u|^{p-2} \nabla u\right)$ occurs in many mathematical models of physical topics including glaciology, nonlinear diffusion and filtration problem (see [4, 17]), power-low materials [14], non-Newtonian fluids [3]. For a discussion of some physical background, see [10]. The nonlinear boundary condition (1.2) describes a flux through the boundary $\partial \Omega$ which depends on the solution itself. For physical motivation of such conditions see for example [16].

Observe that in the particular case $\mu=0$ and $p=2,(1.1)-(1.2)$ becomes linear and it is known as the Steklov problem [7].

2000 Mathematics Subject Classification: 35P30, 35J20, 35J60.

Key words and phrases: $p$-Laplacian operator, principal eigencurve, nonlinear boundary conditions, Sobolev trace embedding. 
Classical Dirichlet problems involving the $p$-Laplacian have been extensively studied by various authors in the cases $\lambda=0$ or $\mu=0$ (cf. e.g. $[1,2,5,10,13,18,19])$. For nonlinear boundary conditions such as (1.2), recently the authors of [8] studied the case of $\mu=0$ and $\varrho$ belonging to some $L^{s}(\partial \Omega)$, not necessarily essentially bounded, with a restrictive condition on its sign.

We set

$$
\begin{aligned}
\mu_{1}(\lambda)=\inf \left\{\|v\|_{1, p}^{p}-\lambda \int_{\partial \Omega} \varrho(x)|v|^{p} d \sigma:\right. & \\
v & \left.\in W^{1, p}(\Omega), \int_{\partial \Omega}|u|^{p} d \sigma=1\right\},
\end{aligned}
$$

where $\|\cdot\|_{1, p}$ denotes the $W^{1, p}(\Omega)$-norm, i.e.,

$$
\|v\|_{1, p}=\left(\|\nabla v\|_{p}^{p}+\|v\|_{p}^{p}\right)^{1 / p}
$$

and $\|\cdot\|_{p}$ is the $L^{p}$-norm, with $\sigma$ being the $(N-1)$-dimensional Lebesgue measure. By the principal (or first) eigencurve of the $p$-Laplacian related to the Sobolev trace embedding, we understand the graph of the map $\mu_{1}$ : $\lambda \mapsto \mu_{1}(\lambda)$ from $\mathbb{R}$ into $\mathbb{R}$. In [12] the simplicity and isolation of the first eigencurve of the Dirichlet $p$-Laplacian was proved by extending a similar result shown by Binding and Huang in [6].

Our purpose is to obtain some results (known for the ordinary Dirichlet $p$-Laplacian) for nonlinear eigenvalue problems where two-parameter eigenvalues appear in the nonlinear boundary condition. We show that $\mu_{1}(\lambda)$ is simple and isolated for any $\lambda \in \mathbb{R}$. Note that to show the simplicity (uniqueness) result, we use a simple convexity argument by remarking that the energy functional associated to problem (1.1)-(1.2) is convex in $u^{p}$ for nonnegative $u$, without using in any way $C^{1}(\Omega)$ and $L^{\infty}(\Omega)$ regularity of the eigenfunctions associated to (1.1)-(1.2). In this respect our procedure is new.

Observe that $\mu_{1}(0)=\lambda_{1}$ is the optimal reciprocal constant of the Sobolev embedding $W^{1, p}(\Omega) \hookrightarrow L^{p}(\partial \Omega)$. For the particular case $\mu=0$ and $\varrho \in$ $L^{s}(\partial \Omega)$ (for a suitable $s$ ), the isolation and simplicity of the first eigenvalue of (1.1)-(1.2) were studied in [8]. The main objective of our work is to extend this result to any $\lambda \in \mathbb{R}$, by using new technical methods.

The rest of the paper is organized as follows. In Section 2, we establish some definitions and preliminaries. In Section 3, we use a variational method to prove the existence of a sequence of eigencurves of (1.1)-(1.2). In Section 4, we prove the simplicity and isolation results for each point of the first eigencurve. Finally, in Section 5, we show some results about variations of the weight as a direct application of the simplicity result. 
2. Definitions. In this paper, all solutions are weak ones, i.e., $u \in$ $W^{1, p}(\Omega)$ is a solution of (1.1)-(1.2) if for all $v \in W^{1, p}(\Omega)$,

$$
\int_{\Omega}|\nabla u|^{p-2} \nabla u \nabla v d x+\int_{\Omega}|u|^{p-2} u v d x=\int_{\partial \Omega}(\lambda \varrho(x)+\mu)|u|^{p-2} u v d \sigma .
$$

If $u \in W^{1, p}(\Omega) \backslash\{0\}$, then $u$ is called an eigenfunction of (1.1)-(1.2) associated to the eigenpair $(\lambda, \mu)$.

Set

$$
\mathcal{M}=\left\{u \in W^{1, p}(\Omega): \int_{\partial \Omega}|u|^{p} d \sigma=1\right\} .
$$

A principal eigenfunction of (1.1)-(1.2) is any eigenfunction $u \in \mathcal{M}, u \geq 0$ a.e. on $\bar{\Omega}$, associated to the pair $\left(\lambda, \mu_{1}(\lambda)\right)$.

Define the following energy functionals on $W^{1, p}(\Omega)$ :

$$
\begin{aligned}
\Phi_{\lambda}(u) & =\frac{1}{p}\|u\|_{1, p}^{p}-\frac{\lambda}{p} \int_{\partial \Omega} \varrho(x)|u|^{p} d \sigma=\frac{1}{p}\|u\|_{1, p}^{p}+\Phi(u), \quad \lambda \in \mathbb{R}, \\
\Psi(u) & =\frac{1}{p} \int_{\partial \Omega}|u|^{p} d \sigma .
\end{aligned}
$$

It is clear that for any $\lambda \in \mathbb{R}$, the solutions of (1.1)-(1.2) are the critical points of $\Phi_{\lambda}$ restricted to $\mathcal{M}$. We shall deal with operators $T$ acting from $W^{1, p}(\Omega)$ into $\left(W^{1, p}(\Omega)\right)^{\prime} . T$ is said to belong to the class $\left(S_{+}\right)$if for any sequence $v_{n}$ weakly convergent to $v$ in $W^{1, p}(\Omega)$ with $\lim _{\sup } \sup _{n \rightarrow \infty}\left\langle T v_{n}, v_{n}-v\right\rangle$ $\leq 0$, it follows that $v_{n} \rightarrow v$ strongly in $W^{1, p}(\Omega)$, where $\left(W^{1, p}(\Omega)\right)^{\prime}$ is the dual of $W^{1, p}(\Omega)$ with respect to the pairing $\langle\cdot, \cdot\rangle$.

3. Existence results. We will use Lyusternik-Schnirelmann theory on $C^{1}$-manifolds (see [19]). It is clear that for any $\lambda \in \mathbb{R}$, the functional $\Phi_{\lambda}$ is even and bounded from below on $\mathcal{M}$. Indeed, if $u \in \mathcal{M}$, then

$$
\Phi_{\lambda}(u) \geq \frac{1}{p}\left(\|u\|_{1, p}^{p}-|\lambda|\|\varrho\|_{\infty, \partial \Omega}\right) .
$$

So

$$
\Phi_{\lambda}(u) \geq \frac{1}{p}\left(\lambda_{1}-|\lambda|\|\varrho\|_{\infty, \partial \Omega}\right)>-\infty,
$$

where $\lambda_{1}=\mu_{1}(0)$ is the reciprocal of the optimal constant in the Sobolev trace embedding $W^{1, p}(\Omega) \hookrightarrow L^{p}(\partial \Omega)$.

By employing the Sobolev trace embedding, we deduce that:

- $\Psi$ and $\Phi$ are weakly continuous,

- $\Psi^{\prime}$ and $\Phi^{\prime}$ are compact.

The following lemma is the key to showing the existence. 
LEMMA 3.1. For any $\lambda \in \mathbb{R}$, we have:

(i) $\left(\Phi_{\lambda}\right)^{\prime}$ maps bounded sets to bounded sets;

(ii) if $u_{n} \rightarrow u$ (weakly) in $W^{1, p}(\Omega)$ and $\left(\Phi_{\lambda}\right)^{\prime}\left(u_{n}\right)$ converges strongly in $\left(W^{1, p}(\Omega)\right)^{\prime}$, then $u_{n} \rightarrow u$ (strongly) in $W^{1, p}(\Omega)$;

(iii) the functional $\Phi_{\lambda}$ satisfies the Palais-Smale condition on $\mathcal{M}$, i.e., for $\left(u_{n}\right)_{n} \subset \mathcal{M}$, if $\Phi_{\lambda}\left(u_{n}\right)$ is bounded and

$$
\left(\Phi_{\lambda}\right)^{\prime}\left(u_{n}\right)-c_{n} \Psi^{\prime}\left(u_{n}\right) \rightarrow 0
$$

with $c_{n}=\left\langle\left(\Phi_{\lambda}\right)^{\prime}\left(u_{n}\right), u_{n}\right\rangle /\left\langle\Psi^{\prime}\left(u_{n}\right), u_{n}\right\rangle$, then $\left(u_{n}\right)_{n}$ has a subsequence convergent in $W^{1, p}(\Omega)$.

Proof. (i) Let $u, v \in W^{1, p}(\Omega)$. Then

$$
\left\langle\left(\Phi_{\lambda}\right)^{\prime}(u), v\right\rangle=\int_{\Omega}|\nabla u|^{p-2} \nabla u \nabla v d x+\int_{\Omega}|u|^{p-2} u v d x+\int_{\partial \Omega} \varrho(x)|u|^{p-2} u v d \sigma .
$$

By Hölder's inequality, we obtain

$$
\begin{aligned}
\left|\left\langle\left(\Phi_{\lambda}\right)^{\prime}(u), v\right\rangle\right| \leq & \left(\int_{\Omega}|\nabla u|^{(p-1) p^{\prime}} d x\right)^{1 / p^{\prime}}\|\nabla v\|_{p}+\left(\int_{\Omega}|u|^{(p-1) p^{\prime}} d x\right)^{1 / p^{\prime}}\|v\|_{p} \\
& +|\lambda|\|\varrho\|_{\infty, \partial \Omega}\left(\int_{\partial \Omega}|u|^{(p-1) p^{\prime}} d \sigma\right)^{1 / p^{\prime}}\|v\|_{p, \partial \Omega} \\
= & \|\nabla u\|_{p}^{p-1}\|\nabla v\|_{p}+\|u\|_{p}^{p-1}\|v\|_{p}+|\lambda|\|\varrho\|_{\infty, \partial \Omega}\|u\|_{p, \partial \Omega}^{p-1}\|v\|_{p, \partial \Omega} .
\end{aligned}
$$

Now, the Sobolev trace embedding $W^{1, p}(\Omega) \hookrightarrow L^{p}(\partial \Omega)$ ensures the existence of a constant $c>0$ such that

$$
\|w\|_{p, \partial \Omega} \leq c\|w\|_{1, p} \quad \text { for any } w \in W^{1, p}(\Omega) .
$$

Hence we deduce that

$$
\left|\left\langle\left(\Phi_{\lambda}\right)^{\prime}(u), v\right\rangle\right| \leq\|\nabla u\|_{p}^{p-1}\|\nabla v\|_{p}+\|u\|_{p}^{p-1}\|v\|_{p}+c^{p}|\lambda|\|\varrho\|_{\infty, \partial \Omega}\|u\|_{1, p}^{p-1}\|v\|_{1, p} .
$$

It is clear that

$$
\|\nabla u\|_{p}^{p-1}\|\nabla v\|_{p}+\|u\|_{p}^{p-1}\|v\|_{p} \leq\|u\|_{1, p}^{p-1}\|v\|_{1, p}
$$

Combining the above inequalities, we conclude that

$$
\mid\left\langle\left(\Phi_{\lambda}\right)^{\prime}(u), v\right| \leq\left(1+c^{p}|\lambda|\|\varrho\|_{\infty, \partial \Omega}\right)\|u\|_{1, p}^{p-1}\|v\|_{1, p}
$$

for any $u, v \in W^{1, p}(\Omega)$. It follows that

$$
\left\|\left(\Phi_{\lambda}\right)^{\prime}(u)\right\| \leq\left(1+c^{p}|\lambda|\|\varrho\|_{\infty, \partial \Omega}\right)\|u\|_{1, p}^{p-1},
$$

where $\|\cdot\|$ denotes the norm of $\left(W^{1, p}(\Omega)\right)^{\prime}$. This implies (i).

(ii) We use condition $\left(S_{+}\right)$as follows. $\left(\Phi_{\lambda}\right)^{\prime}\left(u_{n}\right)$ being strongly convergent to some $f \in\left(W^{1, p}(\Omega)\right)^{\prime}$, by a calculation we have

$$
\left\langle A u_{n}, v\right\rangle=\left\langle-\Delta_{p} u_{n}, v\right\rangle+\int_{\Omega}\left|u_{n}\right|^{p-2} u_{n} v d x+\int_{\partial \Omega}\left|\nabla u_{n}\right|^{p-2} \nabla u_{n} \nu v d \sigma
$$


for any $v \in W^{1, p}(\Omega)$, where $A$ is the operator from $W^{1, p}(\Omega)$ into $\left(W^{1, p}(\Omega)\right)^{\prime}$ defined by

$$
\langle A u, v\rangle=\int_{\Omega}|\nabla u|^{p-2} \nabla u \nabla v d x+\int_{\Omega}|u|^{p-2} u v d x .
$$

This operator satisfies condition $\left(S_{+}\right)$because $-\Delta_{p}$ does (cf. [12]).

If we take $v=u_{n}-u$ in (3.3) we obtain

$$
\begin{aligned}
\left\langle A u_{n}, u_{n}-v\right\rangle= & \left\langle-\Delta_{p} u_{n}, u_{n}-v\right\rangle+\int_{\Omega}\left|u_{n}\right|^{p-2} u_{n}\left(u_{n}-u\right) d x \\
& +\int_{\partial \Omega}\left|\nabla u_{n}\right|^{p-2} \nabla u_{n} \nu\left(u_{n}-u\right) d \sigma .
\end{aligned}
$$

Introducing $\left(\Phi_{\lambda}\right)^{\prime}\left(u_{n}\right)$, we deduce that

$$
\left\langle A u_{n}, u_{n}-u\right\rangle=\left\langle\left(\Phi_{\lambda}\right)^{\prime}\left(u_{n}\right)-f, u_{n}-u\right\rangle+\left\langle f, u_{n}-u\right\rangle-\left\langle\left(\Phi_{\lambda}\right)^{\prime}\left(u_{n}\right), u_{n}-u\right\rangle .
$$

Using the compactness of $\Phi^{\prime}$, we find that as $n \rightarrow \infty$,

$$
\limsup _{n \rightarrow \infty}\left\langle A u_{n}, u_{n}-u\right\rangle \geq 0 \text {. }
$$

Hence $u_{n} \rightarrow u$ strongly in $W^{1, p}(\Omega)$, by condition $\left(S_{+}\right)$.

(iii) From (3.1) we deduce that $\left(u_{n}\right)_{n}$ is bounded in $W^{1, p}(\Omega)$. Thus, without loss of generality, we can assume that $u_{n} \rightarrow u$ (weakly) in $W^{1, p}(\Omega)$ for some $u \in W^{1, p}(\Omega)$. It follows that $\Psi^{\prime}\left(u_{n}\right) \rightarrow \Psi^{\prime}(u)$ in $\left(W^{1, p}(\Omega)\right)^{\prime}$ and $p \Psi(u)=1$, because $p \Psi\left(u_{n}\right)=1$ for all $n \in \mathbb{N}^{*}$. Hence $u \in \mathcal{M}$. Since $\left(u_{n}\right)_{n}$ is bounded, (i) ensures that $\left\{\left(\Phi_{\lambda}\right)^{\prime}\left(u_{n}\right)\right\}$ is bounded. By a calculation we deduce via $(3.2)$ that $\left\{\left(\Phi_{\lambda}\right)^{\prime}\left(u_{n}\right)\right\}$ converges strongly in $\left(W^{1, p}(\Omega)\right)^{\prime}$. Consequently, from (ii) we conclude that $u_{n} \rightarrow u$ (strongly) in $W^{1, p}(\Omega)$.

Set $\Gamma_{k}=\{K \subset \mathcal{M}: K$ symmetric, compact and $\gamma(K)=k\}$, where $\gamma(K)=k$ is the genus of $K$, i.e., the smallest integer $k$ such that there is an odd continuous map from $K$ to $\mathbb{R}^{k} \backslash\{0\}$.

Next, we establish our existence result.

TheOREM 3.1. For any $\lambda \in \mathbb{R}$ and any integer $k \in \mathbb{N}^{*}$,

$$
\mu_{k}(\lambda):=\inf _{K \in \Gamma_{k}} \max _{u \in K} \Phi_{\lambda}(u)
$$

is a critical value of $\Phi_{\lambda}$ restricted to $\mathcal{M}$. More precisely, there exists $u_{k}(\lambda)$ $\in \mathcal{M}$ such that

$$
\mu_{k}(\lambda)=p \Phi_{\lambda}\left(u_{k}(\lambda)\right)=\max _{u \in K} p \Phi_{\lambda}(u)
$$

and $\left(u_{k}(\lambda), \mu_{k}(\lambda)\right)$ is a solution of (1.1)-(1.2). Moreover,

$$
\mu_{k}(\lambda) \rightarrow \infty \quad \text { as } k \rightarrow \infty .
$$

Proof. In view of [19], we need only prove that $\Gamma_{k} \neq \emptyset$ for any $k \in \mathbb{N}^{*}$, and the last assertion. 
Indeed, since $W^{1, p}(\Omega)$ is separable, there exist $\left(e_{i}\right)_{i \geq 1}$ linearly dense in $W^{1, p}(\Omega)$ such that $\operatorname{supp} e_{i} \cap \operatorname{supp} e_{j}=\emptyset$ if $i \neq j$, where supp $e_{i}$ denotes the support of $e_{i}$. We can suppose that $e_{i} \in \mathcal{M}$ (if not we take $e_{i}^{\prime}=e_{i} / p \Psi\left(e_{i}\right)$ ). For $k \in \mathbb{N}^{*}$, define $\mathcal{F}_{k}=\operatorname{span}\left\{e_{1}, \ldots, e_{k}\right\}$. Then $\mathcal{F}_{k}$ is a vector subspace and $\operatorname{dim} \mathcal{F}_{k}=k$. If $v \in \mathcal{F}_{k}$, then there exist $\alpha_{1}, \ldots, \alpha_{k}$ in $\mathbb{R}$ such that $v=\sum_{i=1}^{k} \alpha_{i} e_{i}$. Thus $\Psi(v)=\sum_{i=1}^{k}\left|\alpha_{i}\right|^{p} \Psi\left(e_{i}\right)=p^{-1} \sum_{i=1}^{k}\left|\alpha_{i}\right|^{p}$, because $\Psi\left(e_{i}\right)=1$ for $i=1, \ldots, k$. It follows that the map $v \mapsto(p \Psi(v))^{1 / p}$ is a norm on $\mathcal{F}_{k}$. Hence, there is a constant $c>0$ so that

$$
c\|v\|_{1, p} \leq(p \Psi(v))^{1 / p} \leq \frac{1}{c}\|v\|_{1, p}, \quad \forall v \in \mathcal{F}_{k} .
$$

That is,

$$
c\|v\|_{1, p} \leq\left(\int_{\partial \Omega}|v|^{p} d \sigma\right)^{1 / p} \leq \frac{1}{c}\|v\|_{1, p}, \quad \forall v \in \mathcal{F}_{k} .
$$

This implies that the set

$$
\mathcal{V}=\mathcal{F}_{k} \cap\left\{v \in W^{1, p}(\Omega):\|v\|_{p, \partial \Omega} \leq 1\right\}
$$

is bounded, because $\mathcal{V} \subset B(0,1 / c)=\left\{v \in W^{1, p}:\|v\|_{1, p} \leq 1 / c\right\}$. Moreover $\mathcal{V}$ is a symmetric bounded neighborhood of the origin 0 . Consequently, from Proposition 2.3 of [19], we deduce that $\gamma\left(\mathcal{F}_{k} \cap \mathcal{M}\right)=k$. Then $\mathcal{F}_{k} \cap \mathcal{M} \in \Gamma_{k}$ (because $\mathcal{F}_{k} \cap \mathcal{M}$ is compact, since it equals the boundary of $\mathcal{V}$ ).

To complete the proof, it suffices to show that for any $\lambda \in \mathbb{R}, \mu_{k}(\lambda) \rightarrow \infty$ as $k \rightarrow \infty$. Indeed, let $\left(e_{n}, e_{j}^{*}\right)_{n, j}$ be a biorthogonal system such that $e_{n} \in$ $W^{1, p}(\Omega), e_{j}^{*} \in\left(W^{1, p}(\Omega)\right)^{\prime}$, the $\left(e_{n}\right)_{n}$ are linearly dense in $W^{1, p}(\Omega)$, and the $\left(e_{j}^{*}\right)_{j}$ are total in $\left(W^{1, p}(\Omega)\right)^{\prime}$. For any $k \in \mathbb{N}^{*}$ set

$$
\mathcal{F}_{k-1}^{\perp}=\overline{\operatorname{span}\left(e_{k+1}, e_{k+2}, \ldots\right)} \text {. }
$$

Observe that $K \cap \mathcal{F}_{k-1}^{\perp} \neq \emptyset$ for any $K \in \Gamma_{k}$ (by Proposition 2.3(g) of [19]). Now, we claim that

$$
t_{k}:=\inf _{K \in \Gamma_{k}} \sup _{K \cap \mathcal{F}_{k-1}^{\perp}} p \Phi_{\lambda}(u) \rightarrow \infty \quad \text { as } k \rightarrow \infty .
$$

Indeed, to obtain a contradiction, assume that for $k$ large enough there is $u_{k} \in \mathcal{F}_{k-1}^{\perp}$ with $\int_{\partial \Omega}\left|u_{k}\right|^{p} d \sigma=1$ such that

$$
t_{k} \leq p \Phi_{\lambda}\left(u_{k}\right) \leq M
$$

for some $M>0$ independent of $k$. Then

$$
\left\|u_{k}\right\|_{1, p}^{p}-\lambda \int_{\partial \Omega} \varrho(x)\left|u_{k}\right|^{p} d \sigma \leq M
$$

Hence

$$
\left\|u_{k}\right\|_{1, p}^{p} \leq M+\lambda\|\varrho\|_{\infty, \partial \Omega}<\infty
$$


This implies that $\left(u_{k}\right)_{k}$ is bounded in $W^{1, p}(\Omega)$. Taking a subsequence if necessary, we can suppose that $\left(u_{k}\right)$ converges weakly in $W^{1, p}(\Omega)$ and strongly in $L^{p}(\partial \Omega)$. By our choice of $\mathcal{F}_{k-1}^{\perp}$, we have $u_{k} \rightarrow 0$ in $W^{1, p}(\Omega)$ because $\left\langle e_{n}^{*}, e_{k}\right\rangle=0$ for all $k \geq n$. This contradicts the fact that $\int_{\partial \Omega}\left|u_{k}\right|^{p} d \sigma=1$ for all $k$, and the claim is proved.

Finally, since $\mu_{k}(\lambda) \geq t_{k}$ we conclude that $\mu_{k}(\lambda) \rightarrow \infty$ as $k \rightarrow \infty$, and the proof is complete.

\section{Simplicity and isolation of $\mu_{1}(\lambda)$}

4.1. Simplicity. First, observe that solutions of (1.1)-(1.2), by the wellknown advanced regularity, belong to $C^{1, \alpha}(\bar{\Omega})$ (see $[20]$ ).

LEMMA 4.1. Eigenfunctions $u$ associated to $\mu_{1}(\lambda)$ are either positive or negative in $\Omega$. Moreover if $u \in C^{1, \alpha}(\Omega)$ then $u \neq 0$ in $\bar{\Omega}$.

Proof. Let $u$ be an eigenfunction associated to $\mu_{1}(\lambda)$. Since $\Phi_{\lambda}(|u|) \leq$ $\Phi_{\lambda}(u)$ and $\Psi(|u|)=\Psi(u)$, it follows from (1.3) that $|u|$ is also an eigenfunction associated to $\mu_{1}(\lambda)$. Using Harnack's inequality (cf. [14]), we deduce that $|u|>0$ in $\Omega$. By regularity $u$ is defined in the whole of $\bar{\Omega}$. In fact $|u|>0$ in $\bar{\Omega}$ because $(\partial u / \partial \nu)\left(x_{0}\right)<0$ for any $x_{0} \in \partial \Omega$ with $u\left(x_{0}\right)=0$, by Hopf's Lemma (see [21]).

Theorem 4.1 (Uniqueness). For any $\lambda \in \mathbb{R}, \mu_{1}(\lambda)$ defined by (1.3) is a simple eigenvalue, i.e., the set of eigenfunctions associated to $\left(\lambda, \mu_{1}(\lambda)\right)$ is $\left\{t u_{1}(\lambda): t \in \mathbb{R}\right\}$, where $u_{1}(\lambda)$ denotes the principal eigenfunction associated to $\left(\lambda, \mu_{1}(\lambda)\right)$.

Proof. By Theorem 3.1 it is clear that $\mu_{1}(\lambda)$ is an eigenvalue of the problem (1.1)-(1.2) for any $\lambda \in \mathbb{R}$. Let $u$ and $v$ be two eigenfunctions associated to $\left(\lambda, \mu_{1}(\lambda)\right)$ such that $u, v \in \mathcal{M}$. Thus in virtue of Lemma 4.1 we can assume that $u$ and $v$ are positive.

Note that the mappings $W^{1, p}(\Omega) \ni w \mapsto\|\nabla w\|_{p}^{p}, w \mapsto \int_{\partial \Omega}|w|^{p} d \sigma$ and $w \mapsto \int_{\partial \Omega} \varrho(x)|w|^{p} d \sigma$ are linear functionals in $w^{p}$, for $w^{p} \geq 0$. Hence if we consider

$$
w=\left(\frac{u^{p}+v^{p}}{2}\right)^{1 / p},
$$

then it belongs to $W^{1, p}(\Omega)$ and $\int_{\partial \Omega}|w|^{p} d \sigma=1$. Consequently, $w$ is admissible in the definition of $\mu_{1}(\lambda)$. On the other hand, by the convexity of $\chi \mapsto|\chi|^{p}$ we have the inequalities

$$
\begin{aligned}
\int_{\Omega}|\nabla w|^{p} d x & =\frac{1}{2} \int_{\Omega}\left(\left|u^{p-1} \nabla u+v^{p-1} \nabla v\right|^{p}\left(u^{p}+v^{p}\right)^{1-p}\right) d x \\
& =\frac{1}{2} \int_{\Omega}\left|\frac{u^{p}}{u^{p}+v^{p}} \frac{\nabla u}{u}+\frac{v^{p}}{v^{p}+u^{p}} \frac{\nabla v}{v}\right|^{p}\left(u^{p}+v^{p}\right)^{1-p} d x
\end{aligned}
$$




$$
\begin{aligned}
& \leq \frac{1}{2} \int_{\Omega}\left(\frac{u^{p}}{u^{p}+v^{p}}\left|\frac{\nabla u}{u}\right|^{p}+\frac{v^{p}}{v^{p}+u^{p}}\left|\frac{\nabla v}{v}\right|^{p}\right) d x \\
& \leq \frac{1}{2} \int_{\Omega}\left(|\nabla u|^{p}+|\nabla v|^{p}\right) d x .
\end{aligned}
$$

By the choice of $u$ and $v$, we deduce that

$$
\left|t \frac{\nabla u}{u}+(1-t) \frac{\nabla v}{v}\right|^{p}=t\left|\frac{\nabla u}{u}\right|^{p}+(1-t)\left|\frac{\nabla v}{v}\right|^{p}
$$

with $t=u^{p} /\left(u^{p}+v^{p}\right)$.

Now, we claim that $u=v$ a.e. on $\bar{\Omega}$. Indeed, consider the auxiliary function

$$
F\left(\chi_{1}, \chi_{2}\right)=\left|t \chi_{1}+(1-t) \chi_{2}\right|^{p}-t\left|\chi_{1}\right|^{p}+(1-t)\left|\chi_{2}\right|^{p} .
$$

Since $t \neq 0$, the critical points of $F$ are the solutions of the system

$$
\begin{aligned}
& \frac{\partial F\left(\chi_{1}, \chi_{2}\right)}{\partial \chi_{1}}=p t\left(\left|t \chi_{1}+(1-t) \chi_{2}\right|^{p-2}\left(t \chi_{1}-\left|\chi_{1}\right|^{p-2} \chi_{1}\right)=0\right. \\
& \frac{\partial F\left(\chi_{1}, \chi_{2}\right)}{\partial \chi_{2}}=p(t-1)\left(\left|t \chi_{1}+(1-t) \chi_{2}\right|^{p-2}\left(t \chi_{1}-\left|\chi_{2}\right|^{p-2} \chi_{2}\right)=0 .\right.
\end{aligned}
$$

Thus (4.2)-(4.4) imply that $\left(\chi_{1}=\nabla u / u, \chi_{2}=\nabla v / v\right)$ is a solution of the above system. Therefore

$$
\left|\frac{\nabla u}{u}\right|^{p-2} \frac{\nabla u}{u}=\left|\frac{\nabla v}{v}\right|^{p-2} \frac{\nabla v}{v} .
$$

Hence

$$
\frac{\nabla u}{u}=\frac{\nabla v}{v} \quad \text { a.e. in } \bar{\Omega} \text {. }
$$

This implies easily that $u=c v$ for some positive constant $c$. By normalization we conclude that $c=1$.

REMARK 4.1. Various proofs of the uniqueness result were given in the Dirichlet $p$-Laplacian case by using $C^{1, \alpha}$-regularity and $L^{\infty}$-estimation of the first eigenfunctions and by applying either Picone's identity (cf. [1]) or Díaz-Saá's inequality (cf. [2, 9, 11]) or an abstract inequality (cf. [15]).

\subsection{Isolation}

Proposition 4.1. For any $\lambda \in \mathbb{R}, \mu_{1}(\lambda)$ is the unique eigenvalue associated to $\lambda$, having an eigenfunction not changing its sign on the boundary $\partial \Omega$.

Proof. Fix $\lambda \in \mathbb{R}$ and let $u_{1}(\lambda)$ be the principal eigenfunction associated to $\left(\lambda, \mu_{1}(\lambda)\right)$. Suppose that there exists an eigenfunction $v$ corresponding to a pair $(\lambda, \mu)$ with $v \geq 0$ on $\partial \Omega$ and $v \in \mathcal{M}$. By the Maximum Principle, 
$v>0$ on $\bar{\Omega}$. To simplify the notation, set $u=u_{1}(\lambda)$. For $\varepsilon>0$ small enough, write

$$
\begin{gathered}
u_{\varepsilon}=u+\varepsilon, \quad v_{\varepsilon}=v+\varepsilon, \\
\phi\left(u_{\varepsilon}, v_{\varepsilon}\right)=\frac{u_{\varepsilon}^{p}-v_{\varepsilon}^{p}}{u_{\varepsilon}^{p-1}} .
\end{gathered}
$$

It is clear that $\phi\left(u_{\varepsilon}, v_{\varepsilon}\right) \in W^{1, p}(\Omega)$ and it is an admissible test function in (1.1)-(1.2). Thus we obtain

$$
\begin{aligned}
\int_{\Omega}|\nabla u|^{p-2} \nabla u \nabla \phi\left(u_{\varepsilon}, v_{\varepsilon}\right) d x & +\int_{\Omega} u^{p-1} \phi\left(u_{\varepsilon}, v_{\varepsilon}\right) d x \\
& =\int_{\partial \Omega}\left(\lambda \varrho(x)+\mu_{1}(\lambda)\right) u^{p-1} \phi\left(u_{\varepsilon}, v_{\varepsilon}\right) d \sigma
\end{aligned}
$$

and

$$
\begin{aligned}
\int_{\Omega}|\nabla v|^{p-2} \nabla v \nabla \phi\left(u_{\varepsilon}, v_{\varepsilon}\right) d x+ & \int_{\Omega} v^{p-1} \phi\left(u_{\varepsilon}, v_{\varepsilon}\right) d x \\
& \left.=\int_{\partial \Omega}(\lambda \varrho(x)+\mu)\right) v^{p-1} \phi\left(u_{\varepsilon}, v_{\varepsilon}\right) d \sigma .
\end{aligned}
$$

From (4.7) and (4.8), we deduce by calculation that

$$
\begin{aligned}
\int_{\Omega}|\nabla u|^{p-2} \nabla u \nabla \phi\left(u_{\varepsilon}, v_{\varepsilon}\right) d x+ & \int_{\Omega}|\nabla v|^{p-2} \nabla v \nabla \phi\left(u_{\varepsilon}, v_{\varepsilon}\right) d x \\
& +\int_{\Omega}|v|^{p-2} v \phi\left(u_{\varepsilon}, v_{\varepsilon}\right) d x
\end{aligned}
$$

$$
\begin{aligned}
= & \int_{\partial \Omega} \lambda \varrho(x)\left(\left(\frac{u}{u_{\varepsilon}}\right)^{p-1}-\left(\frac{v}{v_{\varepsilon}}\right)^{p-1}\right)\left(u_{\varepsilon}^{p}-v_{\varepsilon}^{p}\right) d \sigma \\
& +\mu_{1}(\lambda) \int_{\partial \Omega} u^{p-1}\left[u_{\varepsilon}-\left(\frac{v_{\varepsilon}}{u_{\varepsilon}}\right)^{p-1} v_{\varepsilon}\right] d \sigma+\mu \int_{\partial \Omega} u^{p-1}\left[v_{\varepsilon}-\left(\frac{u_{\varepsilon}}{v_{\varepsilon}}\right)^{p-1} u_{\varepsilon}\right] d \sigma .
\end{aligned}
$$

On the other hand, by a long calculation again, we obtain

$$
\nabla \phi\left(u_{\varepsilon}, v_{\varepsilon}\right)=\left\{1+(p-1)\left(\frac{v_{\varepsilon}}{u_{\varepsilon}}\right)^{p}\right\} \nabla u_{\varepsilon}-p\left(\frac{v_{\varepsilon}}{u_{\varepsilon}}\right)^{p-1} \nabla v_{\varepsilon}
$$

and

$$
\begin{aligned}
\int_{\Omega}\left[u^{p-1} \phi\left(u_{\varepsilon}, v_{\varepsilon}\right)+v^{p-1} \phi\left(u_{\varepsilon}, v_{\varepsilon}\right)\right] d x & \\
= & \int_{\Omega}\left[\left(\frac{u}{u_{\varepsilon}}\right)^{p-1}-\left(\frac{v}{v_{\varepsilon}}\right)^{p-1}\right]\left(u_{\varepsilon}^{p}-v_{\varepsilon}^{p}\right) d x .
\end{aligned}
$$


Therefore (4.9), (4.10) and (4.11) yield

$$
\begin{aligned}
& \int_{\Omega}\left[\left\{1+(p-1)\left(\frac{v_{\varepsilon}}{u_{\varepsilon}}\right)^{p}\right\}\left|\nabla u_{\varepsilon}\right|^{p}+\left\{1+(p-1)\left(\frac{u_{\varepsilon}}{v_{\varepsilon}}\right)^{p}\right\}\left|\nabla v_{\varepsilon}\right|^{p}\right] d x \\
& +\int_{\Omega}\left[-p\left(\frac{v_{\varepsilon}}{u_{\varepsilon}}\right)^{p-1}\left|\nabla v_{\varepsilon}\right|^{p-2} \nabla u_{\varepsilon} \nabla v_{\varepsilon}+p\left(\frac{u_{\varepsilon}}{v_{\varepsilon}}\right)^{p-1}\left|\nabla u_{\varepsilon}\right|^{p-2} \nabla u_{\varepsilon} \nabla v_{\varepsilon}\right] d x \\
& =J_{\varepsilon}+K_{\varepsilon}-I_{\varepsilon}
\end{aligned}
$$

with

$$
\begin{aligned}
I_{\varepsilon}= & \int_{\Omega}\left(\left(\frac{u}{u_{\varepsilon}}\right)^{p-1}-\left(\frac{v}{v_{\varepsilon}}\right)^{p-1}\right)\left(u_{\varepsilon}^{p}-v_{\varepsilon}^{p}\right) d x \\
J_{\varepsilon}= & \lambda \int_{\partial \Omega} \varrho(x)\left(\left(\frac{u}{u+\varepsilon}\right)^{p-1}-\left(\frac{v}{v+\varepsilon}\right)^{p-1}\right)\left(u_{\varepsilon}^{p}-v_{\varepsilon}^{p}\right) d \sigma \\
K_{\varepsilon}= & \mu_{1}(\lambda) \int_{\partial \Omega} u^{p-1}\left[u_{\varepsilon}-\left(\frac{v_{\varepsilon}}{u_{\varepsilon}}\right)^{p-1} v_{\varepsilon}\right] d \sigma \\
& +\mu \int_{\partial \Omega} u^{p-1}\left[v_{\varepsilon}-\left(\frac{u_{\varepsilon}}{v_{\varepsilon}}\right)^{p-1} u_{\varepsilon}\right] d \sigma .
\end{aligned}
$$

It is clear that $I_{\varepsilon} \geq 0$. Now, thanks to the inequalities of Lindqvist [15], we can distinguish two cases according to the value of $p$.

CASE 1: $p \geq 2$. From (4.12) we have

$$
J_{\varepsilon}+K_{\varepsilon} \geq \frac{1}{2^{p-2}-1} \int_{\Omega}\left(\frac{1}{(u+1)^{p}}+\frac{1}{(v+1)^{p}}\right)|u \nabla v-v \nabla u|^{p} d x \geq 0 .
$$

CAse 2: $1<p<2$. Then

$$
J_{\varepsilon}+K_{\varepsilon} \geq c(p) \int_{\Omega} \frac{u v\left(u^{p}+v^{p}\right)}{(v|\nabla u|+u|\nabla v|+1)^{2-p}}|u \nabla v-v \nabla u|^{2} d x \geq 0
$$

where the constant $c(p)>0$ is independent of $u, v, \lambda$ and $\mu_{1}(\lambda)$.

The Dominated Convergence Theorem implies that

$$
\lim _{\varepsilon \rightarrow 0^{+}} J_{\varepsilon}=\lim _{\varepsilon \rightarrow 0^{+}} K_{\varepsilon}=\left(\mu_{1}(\lambda)-\mu\right) \int_{\partial \Omega}\left(u^{p}-v^{p}\right) d \sigma=0,
$$

because

$$
\int_{\partial \Omega} u^{p} d \sigma=\int_{\partial \Omega} v^{p} d \sigma=1
$$

Now, letting $\varepsilon \rightarrow 0^{+}$in (4.16) and (4.17), we arrive at

$$
u \nabla v=v \nabla u \quad \text { a.e. on } \Omega \text {. }
$$


Thus

$$
\nabla\left(\frac{u}{v}\right)=0 \quad \text { a.e. on } \Omega .
$$

Hence, there exists $t>0$ such that $u=t v$ a.e. on $\Omega$. By continuity $u=v$ a.e. in $\bar{\Omega}$; and by the normalization (4.18) we deduce that $t=1$ and $u=v$ a.e. on $\partial \Omega$. This implies that $u=v$ a.e. on $\bar{\Omega}$. Finally, we conclude that $\mu=\mu_{1}(\lambda)$.

Remark 4.2. We can also show Proposition 4.1 by using Picone's identity. A similar result was given in [8] in the particular case $\lambda=0$.

Corollary 4.1. For any $\lambda \in \mathbb{R}$, if $u$ is an eigenfunction associated to a pair $(\lambda, \mu)$ with $\mu \neq \mu_{1}(\lambda)$, then $u$ changes its sign on the boundary $\partial \Omega$. Moreover,

$$
\min \left(\left|\partial \Omega^{-}\right|,\left|\partial \Omega^{+}\right|\right) \geq c_{p^{*}}^{-N}\left(|\lambda|\|\varrho\|_{\infty, \partial \Omega}+|\mu|\right)^{-\eta},
$$

where $\eta=N / p$ if $1<p<N$ and $\eta=2$ if $p>N, c_{p^{*}}$ is the best constant in the Sobolev trace embedding $W^{1, p}(\Omega) \hookrightarrow L^{p^{*}}(\partial \Omega)$, and $\left|\partial \Omega^{ \pm}\right|$denotes the $(N-1)$-dimensional measure of $\partial \Omega^{ \pm}$. Here $p^{*}=p(N-1) /(N-p)$ is the critical Sobolev exponent and $\partial \Omega^{ \pm}=\{x \in \bar{\Omega}: u(x) \gtrless 0\}$.

Proof. Set $u^{+}=\max (u, 0)$ and $u^{-}=\max (-u, 0)$. It follows from (2.1), where we put $v=u^{-}$, that

$$
\int_{\Omega}\left|\nabla u^{-}\right|^{p} d x+\int_{\Omega}\left|u^{-}\right|^{p} d x=\int_{\partial \Omega}(\lambda \varrho(x)+\mu)\left|u^{-}\right|^{p} d \sigma .
$$

Thus

$$
\begin{aligned}
\left\|u^{-}\right\|_{1, p} & \leq\left(|\lambda|\|\varrho\|_{\infty, \partial \Omega}+|\mu|\right) \int_{\partial \Omega^{-}}\left|u^{-}\right|^{p} d \sigma \\
& \leq\left(|\lambda|\|\varrho\|_{\infty, \partial \Omega}+|\mu|\right)\left|\partial \Omega^{-}\right|^{p / N}\left(\int_{\partial \Omega}\left|u^{-}\right|^{p^{*}}\right)^{p / p^{*}} .
\end{aligned}
$$

By the Sobolev embedding $W^{1, p}(\partial \Omega) \hookrightarrow L^{p^{*}}(\partial \Omega)$, we deduce that

$$
\left|\partial \Omega^{-}\right| \geq c_{p^{*}}^{-N}\left(|\lambda|\|\varrho\|_{\infty, \partial \Omega}+|\mu|\right)^{-\eta}
$$

The same holds for $\partial \Omega^{+}$by taking $v=u^{+}$in (2.1). Hence the estimate (4.19) follows.

REMARKS 4.1. (i) The right-hand side of (4.19) is positive because $\varrho \not \equiv 0$ and if $\lambda=0$ then $\mu$ is an eigenvalue of the $p$-Laplacian related to the trace embedding, so $\mu-\lambda_{1}>0$, where $\lambda_{1}$ is the first eigenvalue of (1.1) $-(1.2)$ in the case $\lambda=0$.

(ii) An easy consequence of Corollary 4.1 is that the number of nodal components of each eigenfunction of (1.1)-(1.2) is finite. 
Using Proposition 4.1 and Corollary 4.1, we can state the following important result.

TheOrem 4.2. For any $\lambda \in \mathbb{R}, \mu_{1}(\lambda)$ is isolated.

5. Variations of the weight. Let $\mu_{1}(\lambda)=\mu_{1}(\varrho)$ and $u_{1}(\lambda)=u_{1}(\varrho)$ (to indicate the dependence on the weight $\varrho$ ).

THEOREM 5.1. For any $\lambda \in \mathbb{R}$, if $\left(\varrho_{k}\right)_{k}$ is a sequence in $L^{\infty}(\partial \Omega)$ such that $\varrho_{k}$ converges to $\varrho$ in $L^{\infty}(\partial \Omega)$ with $\varrho \neq \equiv 0$, then

$$
\begin{gathered}
\lim _{k \rightarrow \infty} \mu_{1}\left(\varrho_{k}\right)=\mu_{1}(\varrho), \\
\lim _{k \rightarrow \infty}\left\|u_{1}\left(\varrho_{k}\right)-u_{1}(\varrho)\right\|_{1, p}^{p}=0 .
\end{gathered}
$$

Proof. If $\lambda=0$, the result is evident because $\mu_{1}\left(\varrho_{k}\right)=\mu_{1}(\varrho)$ for all $k \in \mathbb{N}^{*}$. If $\lambda \neq 0$, then for $v \in \mathcal{M}$,

$$
\left.\left|\lambda \int_{\partial \Omega}\left(\varrho_{k}-\varrho\right)\right| v\right|^{p} d \sigma|\leq| \lambda \mid\left\|\varrho_{k}-\varrho\right\|_{\infty, \partial \Omega} .
$$

By the convergence of $\varrho_{k}$ to $\varrho$ in $L^{\infty}(\partial \Omega)$, for every $\varepsilon>0$ there exists $k_{\varepsilon} \in \mathbb{N}$ such that for all $k \geq k_{\varepsilon}$,

$$
\left.\left|\lambda \int_{\partial \Omega}\left(\varrho_{k}-\varrho\right)\right| v\right|^{p} d \sigma|\leq| \lambda \mid \frac{\varepsilon}{|\lambda|}=\varepsilon
$$

This implies that

$$
\begin{gathered}
\lambda \int_{\partial \Omega} \varrho|v|^{p} d \sigma \leq \varepsilon+\lambda \int_{\partial \Omega} \varrho_{k}|v|^{p} d \sigma, \\
\lambda \int_{\partial \Omega} \varrho_{k}|v|^{p} d \sigma \leq \varepsilon+\lambda \int_{\partial \Omega} \varrho|v|^{p} d \sigma,
\end{gathered}
$$

for any $v \in \mathcal{M}, \varepsilon>0$ and $k \geq k_{\varepsilon}$.

On the other hand, we have $\varrho \not \equiv 0$. We take $k_{\varepsilon}$ large enough so that $\varrho_{k} \not \equiv 0$. Thus

$$
\mu_{1}\left(\varrho_{k}\right) \leq\|v\|_{1, p}^{p}-\lambda \int_{\partial \Omega} \varrho_{k}|v|^{p} d \sigma .
$$

Combining with (5.3) and (5.4), we obtain

$$
\mu_{1}\left(\varrho_{k}\right) \leq\|v\|_{1, p}^{p}-\lambda \int_{\partial \Omega} \varrho|v|^{p} d \sigma+\varepsilon
$$

Passing to the infimum over $v \in \mathcal{M}$, we find

$$
\mu_{1}\left(\varrho_{k}\right) \leq \mu_{1}(\varrho)+\varepsilon, \quad \mu_{1}(\varrho) \leq \mu_{1}\left(\varrho_{k}\right)+\varepsilon, \quad \forall \varepsilon>0 \forall k>k_{\varepsilon} .
$$

Hence, we obtain the convergence (5.1). 
For the strong convergence (5.2) we argue as follows. For $k$ large enough, we have $\varrho_{k} \not \equiv 0$ and

$$
\mu_{1}\left(\varrho_{k}\right)=\left\|u_{1}\left(\varrho_{k}\right)\right\|_{1, p}^{p}-\lambda \int_{\partial \Omega} \varrho_{k}\left(u_{1}\left(\varrho_{k}\right)\right)^{p} d \sigma
$$

Thus

$$
\left\|u_{1}\left(\varrho_{k}\right)\right\|_{1, p}^{p} \leq\left|\mu_{1}\left(\varrho_{k}\right)\right|+|\lambda|\left\|\varrho_{k}\right\|_{\infty, \partial \Omega} .
$$

From (5.1) and the convergence of $\varrho_{k}$ to $\varrho$ in $L^{\infty}(\partial \Omega)$, we deduce that $\left(u_{1}\left(\varrho_{k}\right)\right)_{k}$ is a bounded sequence in $W^{1, p}(\Omega)$. Since $W^{1, p}(\Omega)$ is reflexive and compactly embedded in $L^{p}(\partial \Omega)$ we can extract a subsequence of $\left(u_{1}\left(\varrho_{k}\right)\right)_{k}$, again labelled by $k$, such that $u_{1}\left(\varrho_{k}\right) \rightarrow u$ (weakly) in $W^{1, p}(\Omega)$ and $u_{1}\left(\varrho_{k}\right) \rightarrow$ $u$ (strongly) in $L^{p}(\partial \Omega)$ as $k \rightarrow \infty$. We can also suppose that $u_{1}\left(\varrho_{k}\right) \rightarrow u$ in $L^{p}(\Omega)$. Passing to a subsequence if necessary, we can assume that $u_{1}\left(\varrho_{k}\right) \rightarrow u$ a.e. in $\bar{\Omega}$. Thus $u \geq 0$ a.e. in $\bar{\Omega}$. We will prove that $u \equiv u_{1}(\varrho)$. To do this, using the Dominated Convergence Theorem in $\partial \Omega$, we deduce that

$$
\int_{\partial \Omega} \varrho_{k}\left(u_{1}\left(\varrho_{k}\right)\right)^{p} d \sigma \rightarrow \int_{\partial \Omega} \varrho u^{p} d \sigma
$$

as $k \rightarrow \infty$. By (5.5), (5.1) and the lower weak semicontinuity of the norm we obtain

$$
\|u\|_{1, p}^{p} \leq \mu_{1}(\varrho)+\lambda \int_{\partial \Omega} \varrho u^{p} d \sigma .
$$

The normalization $\int_{\partial \Omega} u^{p} d \sigma=1$ is proved. Moreover, $u \geq 0$ a.e. in $\bar{\Omega}$, because $u_{1}\left(\varrho_{k}\right)>0$ in $\bar{\Omega}$. Thus $u$ is an admissible function in the variational definition of $\mu_{1}(\lambda)$. So

This and (5.6) yield

$$
\mu_{1}(\lambda) \leq\|u\|_{1, p}^{p}-\lambda \int_{\partial \Omega} \varrho u^{p} d \sigma .
$$

$$
\mu_{1}(\varrho)=\|u\|_{1, p}^{p}-\lambda \int_{\partial \Omega} \varrho u^{p} d \sigma .
$$

By the uniqueness of the principal eigenfunction associated to $\mu_{1}(\lambda)$, we must have $u \equiv u_{1}(\varrho)$. Consequently, the limit function $u_{1}(\varrho)$ is independent of the choice of the (sub)sequence. Hence, $u_{1}\left(\varrho_{k}\right)$ converges to $u_{1}(\varrho)$ at least in $L^{p}(\partial \Omega)$ and in $L^{p}(\Omega)$. To complete the proof of (5.2), it suffices to use Clarkson's inequalities related to uniform convexity of $W^{1, p}(\Omega)$. For this we distinguish two cases.

CASE 1: $p \geq 2$. We have

$$
\begin{aligned}
\int_{\Omega}\left|\frac{\nabla u_{1}\left(\varrho_{k}\right)-\nabla u_{1}(\varrho)}{2}\right|^{p} d x+\int_{\Omega} & \left|\frac{\nabla u_{1}\left(\varrho_{k}\right)+\nabla u_{1}(\varrho)}{2}\right|^{p} d x \\
& \leq \frac{1}{2} \int_{\Omega}\left|\nabla u_{1}\left(\varrho_{k}\right)\right|^{p} d x+\frac{1}{2} \int_{\Omega}\left|\nabla u_{1}(\varrho)\right|^{p} d x
\end{aligned}
$$


and

$$
\begin{aligned}
\mu_{1}\left(\varrho_{k}\right) \int_{\partial \Omega}\left(\frac{u_{1}\left(\varrho_{k}\right)+u_{1}(\varrho)}{2}\right)^{p} d \sigma \leq & \int_{\Omega}\left|\frac{\nabla u_{1}\left(\varrho_{k}\right)+\nabla u_{1}(\varrho)}{2}\right|^{p} d x \\
& -\lambda \int_{\partial \Omega} \varrho_{k}\left(\frac{u_{1}\left(\varrho_{k}\right)+u_{1}(\varrho)}{2}\right)^{p} d \sigma .
\end{aligned}
$$

Moreover

$$
\int_{\Omega}\left|\frac{u_{1}\left(\varrho_{k}\right)-u_{1}(\varrho)}{2}\right|^{p} d x \leq \int_{\Omega}\left|\frac{u_{1}\left(\varrho_{k}\right)+u_{1}(\varrho)}{2}\right|^{p} d x+\frac{1}{2}\left\|u_{1}\left(\varrho_{k}\right)\right\|_{p}^{p}+\frac{1}{2}\left\|u_{1}(\varrho)\right\|_{p}^{p} \text {. }
$$

Hence

$$
\begin{aligned}
\| u_{1}\left(\varrho_{k}\right) & -u_{1}(\varrho) \|_{1, p}^{p} \\
\leq & -\mu_{1}\left(\varrho_{k}\right) \int_{\partial \Omega}\left(\frac{u_{1}\left(\varrho_{k}\right)+u_{1}(\varrho)}{2}\right)^{p} d \sigma-\lambda \int_{\partial \Omega} \varrho_{k}\left(\frac{u_{1}\left(\varrho_{k}\right)+u_{1}(\varrho)}{2}\right)^{p} d \sigma \\
& +\frac{1}{2}\left(\mu_{1}\left(\varrho_{k}\right)-\lambda \int_{\partial \Omega} \varrho_{k}(x) u_{1}\left(\varrho_{k}\right) d \sigma\right)+\frac{1}{2}\left(\mu_{1}(\varrho)-\lambda \int_{\partial \Omega} \varrho u_{1}^{p} d \sigma\right) .
\end{aligned}
$$

Then, by using the Dominated Convergence Theorem we deduce that

$$
\limsup _{k \rightarrow \infty}\left\|u_{1}\left(\varrho_{k}\right)-u_{1}(\varrho)\right\|_{1, p}^{p}=0 \text {. }
$$

CASE 2: $1<p<2$. In this case, we have

$$
\begin{gathered}
\left\{\int_{\Omega}\left|\frac{\nabla u_{1}\left(\varrho_{k}\right)-\nabla u_{1}(\varrho)}{2}\right|^{p} d x\right\}^{1 /(p-1)}+\left\{\int_{\Omega}\left|\frac{\nabla u_{1}\left(\varrho_{k}\right)+\nabla u_{1}(\varrho)}{2}\right|^{p} d x\right\}^{1 /(p-1)} \\
\leq\left\{\frac{1}{2} \int_{\Omega}\left|\nabla u_{1}\left(\varrho_{k}\right)\right|^{p} d x+\frac{1}{2} \int_{\Omega}\left|\nabla u_{1}(\varrho)\right|^{p} d x\right\}^{1 /(p-1)}
\end{gathered}
$$

and

$$
\begin{aligned}
\mu_{1}\left(\varrho_{k}\right) \int_{\partial \Omega}\left(\frac{u_{1}\left(\varrho_{k}\right)+u_{1}(\varrho)}{2}\right)^{p} d \sigma \leq & \int_{\Omega}\left|\frac{\nabla u_{1}\left(\varrho_{k}\right)+\nabla u_{1}(\varrho)}{2}\right|^{p} \\
& -\lambda \int_{\partial \Omega} \varrho_{k}\left(\frac{u_{1}\left(\varrho_{k}\right)+u_{1}(\varrho)}{2}\right)^{p} d \sigma .
\end{aligned}
$$

Hence, by definitions of $\mu_{1}\left(\varrho_{k}\right)$ and $\mu_{1}(\varrho)$, and the second Clarkson inequality we obtain the convergence (5.2).

COROLlary 5.1. For any bounded domain $\Omega$, the function $\lambda \mapsto \mu_{1}(\lambda)$ is differentiable on $\mathbb{R}$ and the function $\lambda \mapsto u(\lambda)$ is continuous from $\mathbb{R}$ into $W^{1, p}(\Omega)$. More precisely

$$
\mu_{1}^{\prime}\left(\lambda_{0}\right)=-\int_{\partial \Omega} \varrho(x)\left(u_{1}\left(\lambda_{0}\right)\right)^{p} d \sigma, \quad \forall \lambda_{0} \in \mathbb{R} .
$$


Proof. Denote by $\mu_{1}(\lambda, \varrho)$ the principal eigenvalue associated with $\lambda$ and the weight $\varrho$ and by $u_{1}(\lambda, \varrho)$ the corresponding principal eigenfunction. Suppose that $\lambda_{k} \rightarrow \lambda_{0}$ in $\mathbb{R}$; then $h_{k}=\lambda_{k} \varrho \rightarrow \lambda_{0} \varrho=h$ in $L^{\infty}(\partial \Omega)$. From Theorem 5.1 we deduce that

$$
\mu_{1}\left(\lambda_{k}\right)=\mu_{1}\left(1, h_{k}\right) \rightarrow \mu_{1}(1, h)=\mu_{1}\left(\lambda_{0}\right)
$$

and

$$
u_{1}\left(\lambda_{k}\right)=u_{1}\left(1, h_{k}\right) \rightarrow u_{1}(1, h)=u_{1}\left(\lambda_{0}\right) \quad \text { in } W^{1, p}(\Omega) .
$$

For the differentiability, it suffices to use the variational characterization of $\mu_{1}(\lambda)$ and of $\mu_{1}\left(\lambda_{0}\right)$, so that we have

$$
\left(\lambda-\lambda_{0}\right) \int_{\partial \Omega} \varrho(x)\left(u_{1}(\lambda)\right)^{p} d \sigma \leq \mu_{1}(\lambda)-\mu_{1}\left(\lambda_{0}\right) \leq\left(\lambda_{0}-\lambda\right) \int_{\partial \Omega}\left(u_{1}\left(\lambda_{0}\right)\right)^{p} d \sigma
$$

for any $\lambda, \lambda_{0} \in \mathbb{R}$.

\section{References}

[1] W. Allegretto and Y. X. Huang, A Picone's identity for the p-Laplacian and applications, Nonlinear Anal. 32 (1998), 819-830.

[2] A. Anane, Simplicité et isolation de la première valeur propre du p-Laplacien, C. R. Acad. Sci. Paris 305 (1987), 725-728.

[3] C. Atkinson and C. R. Champion, Some boundary-value problems for the equation $\nabla \cdot\left(|\nabla \phi|^{N} \nabla \phi\right)=0$, Quart. J. Mech. Appl. Math. 37 (1984), 401-419.

[4] C. Atkinson and C. W. Jones, Similarity solutions in some non-linear diffusion problems and in boundary-layer flow of a pseudo-plastic fluid, ibid. 27 (1974), 193211.

[5] G. Barles, Remarks on uniqueness results for the first eigenvalue of the p-Laplacian, Ann. Fac. Sci. Toulouse 9 (1988), 65-75.

[6] P. A. Binding and Y. X. Huang, The principal eigencurve for $p$-Laplacian, Differential Integral Equations 8 (1995), 405-415.

[7] I. Babuška and J. Osborn, Eigenvalue problems, in: Handbook of Numerical Analysis, Vol. II, North-Holland, Amsterdam, 1991, 641-787.

[8] J. Fernández Bonder and J. D. Rossi, A nonlinear eigenvalue problem with indefinite weights related to Sobolev trace embedding, Publ. Mat. 46 (2002), 221-235.

[9] M. Cuesta, Eigenvalue problems for the p-Laplacian with indefinite weights, Electronic J. Differential Equations 2001, No. 33, 9 pp.

[10] J. I. Díaz, Nonlinear Partial Differential Equations and Free Boundaries, Vol. I, Elliptic Equations, Pitman, London, 1985.

[11] J. I. Díaz et J. E. Saá, Existence et unicité de solutions positives pour certaines équations elliptiques quasilinéaires, C. R. Acad. Sci. Paris Sér. I Math. 305 (1987), $521-524$.

[12] A. El Khalil and A. Touzani, On the first eigencurve of the p-Laplacian, in: Partial Differential Equations, Lecture Notes in Pure and Appl. Math. 229, Dekker, 2002, 195-205.

[13] J. P. García Azorero and I. Peral Alonso, Existence and nonuniqueness for the pLaplacian, Nonlinear eigenvalues, Comm. Partial Differential Equations 12 (1987), 1389-1430. 
[14] D. Gilbarg and N. Trudinger, Elliptic Partial Differential Equations of Second Order, Springer, Berlin, 1983.

[15] P. Lindqvist, On the equation $\operatorname{div}\left(|\nabla u|^{p-2} \nabla u\right)+\lambda|u|^{p-2} u=0$, Proc. Amer. Math. Soc. 109 (1990), 157-164.

[16] C. V. Pao, Nonlinear Parabolic and Elliptic Equations, Plenum Press, New York, 1992.

[17] J. R. Philip, n-diffusion, Austral. J. Phys. 14 (1961), 1-13.

[18] N. M. Stavrakakis and N. B. Zographopoulos, Existence results for quasilinear elliptic systems in $\mathbb{R}^{N}$, Electronic J. Differental Equations 1999, No. 39, 15 pp.

[19] A. Szulkin, Ljusternik-Schnirelmann theory on $C^{1}$-manifolds, Ann. Inst. H. Poincaré Anal. Non Linéaire 5 (1988), 119-139.

[20] P. Tolksdorf, Regularity for a more general class of quasilinear elliptic equations, J. Differential Equations 51 (1983), 126-150.

[21] J. L. Vázquez, A strong maximum principle for some quasilinear elliptic equations, Appl. Math. Optim. 12 (1984), 191-202.

Département de Mathématiques \& Génie Industriel

École Polytechnique, Montréal

Montréal (QC) H3C 3A7

Canada

E-mail: abdelouahed.el-khalil@polymtl.ca
Departement of Mathematics Faculty of Sciences Dhar-Mahraz P.O. Box 1796

Atlas, Fez 30000, Morocco E-mail: m_ouanan@hotmail.com

Received on 4.7.2003;

revised version on 12.7 .2004 